Check for updates

Cite this: RSC Adv., 2018, 8, 37028

Received 10th August 2018

Accepted 8th October 2018

DOI: $10.1039 / c 8 r a 06732 \mathrm{e}$

rsc.li/rsc-advances

\section{A sensitive fluorescent sensor for the detection of trace water in organic solvents based on carbon quantum dots with yellow fluorescence $\uparrow$}

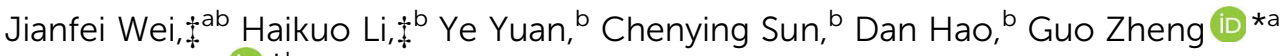 \\ and Rui Wang (D) *b
}

\begin{abstract}
The quantitative analysis of trace water in organic solvents has always been a research hotspot, and it is still in the development stage and needs to be continuously developed. In this study, a facile and rapid approach was developed for the preparation of carbon quantum dots (CQDs) with yellow fluorescence emission and ultrahigh absolute fluorescence quantum yields $(92.6 \%)$. Compared to traditional organic fluorescent molecules, the preparation of CQDs is simpler, faster and more environmentally friendly. It is found that the fluorescent properties of CQDs are excellent in organic solvents and could be quenched by trace water, which makes them a promising material used without any modification for the detection of water in organic solvents. As a result, the as-prepared CQDs were adopted as fluorescent probes for the detection of water in organic solvents (ethanol, tetrahydrofuran, and 1,4-dioxane). The limit of detection was as low as $0.01 \%$. To the best of our knowledge, this is the first time that CQDs have been used as water sensing fluorescent probes in organic solvents. The possible mechanism for trace water detection of the as-prepared CQDs in organic solvents is attributed to the specific water-fluorophore interaction and partially to the increase in polarity of the solvent caused by an increase in water concentration.
\end{abstract}

\section{Introduction}

The detection of trace water in organic solvents is an important analytical tool in several fields of industry and chemistry. ${ }^{1-7}$ The existence of water may cause hydrolysis of organic solvents and increase the formation of oxidation products during storage of organic solvents. ${ }^{8-14}$ At present, water content is usually measured by a classic Karl-Fisher titration. However, this method also has some flaws such as rigorous sample manipulation, the use of toxic reagents, and the need for specialized equipment. ${ }^{15-17}$ Nowadays, fluorescent sensors for the detection of trace water are particularly attractive on account of their selectivity, and high sensitivity, as well as their inexpensive, quick, easy and nondestructive fabrication. Therefore, fluorescent sensors are ideal

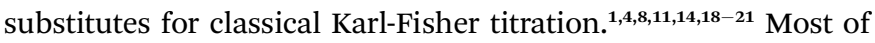
the fluorescent sensors currently developed for the detection of water in organic solvents are based on organic fluorescent dyes and fluorescent conjugated polymers. ${ }^{3, \mathbf{8 , 1 1 , 1 3 , 1 5}}$ Yang et al. reported

${ }^{a}$ School of Material Science and Engineering, Tianjin Polytechnic University, No. 399 BinShuiXi Road, Xiqing District, Tianjin, P. R. China

${ }^{b}$ School of Material Science and Engineering, Beijing Institute of Fashion Technology, No. A2, East Yinghua Street, Chaoyang District, Beijing, P. R. China. E-mail: clywangrui@bift.edu.cn; Tel: +86-10-64288279

$\dagger$ Electronic supplementary information (ESI) available. See DOI: 10.1039/c8ra06732e

$\$$ Jianfei Wei and Haikuo Li contribute equally to this work. several fluorescence water probes based on merocyanine dyes, fluorescein derivative, 8-hydroxyquinoline derivative, merocyanine dyes and a phenol-indole dye. ${ }^{17}$ However, organic fluorescent dyes also have disadvantages such as difficulty in synthesis, long preparation period, certain toxicity of the dye itself, and environmental pollution. Fluorescent carbon quantum dots (CQDs) are a new type of fluorescent carbon nanocomposites, which have many advantages over other organic fluorescent materials, such as less affected by the polarity of the solution, lower biological toxicity, narrow and tunable spectra, longer fluorescence lifetime, broad excitation spectra, resistance of photobleaching and higher molar extinction over the organic fluorescent materials. ${ }^{22-58}$ In this study, a facile and rapid approach was developed for the preparation of carbon quantum dots (CQDs) with yellow fluorescence emission. Compared to traditional organic fluorescent molecules, the preparation of CQDs is simpler, faster and more environment-friendly. It is found that the fluorescent properties of CQDs are excellent in organic solvents and could be quenched by trace water, which makes them a promising material used without any modification for the detection of water in organic solvents. As a result, the asprepared CQDs were adopted as fluorescent probe for the detection of water in organic solvents (ethanol, tetrahydrofuran, 1,4-dioxane). To the best of our knowledge, this is the first time that CQDs have been used as water sensing fluorescent probes in organic solvents. The possible mechanism for trace water detection of the as-prepared CQDs in organic solvents is 
attributed to the specific water-fluorophore interaction and partially to the increase in polarity of the solvent caused by the increase of water concentration.

\section{Materials and methods}

\subsection{Materials}

Sodium citrate, carbamide, methylbenzene and cobalt chloride were obtained from Sinopharm Chemical Reagent Co. Ltd. All of the reagents are analytical grade, which can be used without further purification.

Ethanol absolute (water content $\leq 0.005 \%$, anhydrous level, E130059), tetrahydrofuran ( $\geq 99.9 \%$, anhydrous level, T120775), and 1,4-dioxane (HPLC, $\geq 99.5 \%$, D116159) were purchased from Sigma-Aldrich. All the above solvents were dried with activated $3 \AA$ A molecular sieves.

\subsection{Apparatus}

Transmission Electron Microscopy (TEM) (Model JEM-2100F, 200 $\mathrm{kV}$, Japan Electron Optics Laboratory Ltd.) was adopted to characterize the morphology and size distribution of the as-prepared carbon quantum dots. X-ray Photoelectron Spectroscopy (XPS) analysis was performed using a Thermo Fisher Scientific Escalab 250XI spectrometer with a monochromatic X-ray source $\mathrm{Al} \mathrm{K \alpha}$ excitation $(1486.6 \mathrm{eV})$. The fluorescence excitation and emission spectra, the UV-vis absorption spectra, as well as the quantum yield were measured using the multifunctional fluorescence spectrophotometer (FS5) from Techcomp (China) Ltd. The samples were put in a quartz fluorescence cuvette with $10 \mathrm{~mm}$ optical path length. The samples were excited by the light with wavelength of $440 \mathrm{~nm}$, and the emission spectrum was recorded in the range of 480 to $680 \mathrm{~nm}$. The excitation and emission slit width were fixed at $1 \mathrm{~nm}$ and $2 \mathrm{~nm}$, respectively.

\subsection{Preparation of carbon quantum dots of yellow fluorescence}

In this study, carbon quantum dots were prepared by solvothermal method with sodium citrate, carbamide and cobalt chloride as precursors, while methylbenzene was adopted as solvent. First of all, $0.5882 \mathrm{~g}(0.0020 \mathrm{~mole})$ trisodium citrate dehydrate, $0.2402 \mathrm{~g}$ ( 0.0040 mole) carbamide, $0.7139 \mathrm{~g}(0.0030$ mole) cobalt chloride and $20 \mathrm{~mL}$ methylbenzene were added in a $50 \mathrm{~mL}$ Teflon-lined stainless steel autoclave. Then, the autoclave was placed in a drying oven for 24 hours, when the temperature of the oven is fixed at $180{ }^{\circ} \mathrm{C}$. After that, the asprepared product was purified by filtration using cylindrical filtration membrane filter $(0.22 \mu \mathrm{m})$ and dialysis using regenerated cellulose dialysis membranes with molecular weight cutoffs of $1 \mathrm{kDa}$ (Spectra/Por® 7, 132105). Finally, $1 \mathrm{~mL}$ solution was dried in the lyophilizers at about fifty degrees below zero and dispersed in $3 \mathrm{~mL}$ organic solvents (such as ethyl alcohol, tetrahydrofuran, and dioxane) for further use.

\subsection{Detect moisture content in organic solvents}

The as-prepared CQDS ( $3 \mu \mathrm{L})$ was diluted to $3 \mathrm{~mL}$ corresponding organic solvent and the fluorescent emission spectra excited by the light with wavelength of $440 \mathrm{~nm}$ was recorded in the range of $480 \mathrm{~nm}$ to $680 \mathrm{~nm}$. The fluorescent intensity at $570 \mathrm{~nm}$ was defined as the $F_{0}$. After that, deionized water was added into the above solution, and the fluorescent emission spectra were measured again after 1 minute. The fluorescent intensity at $570 \mathrm{~nm}$ was defined as the $F_{t}$. The change of fluorescent intensity $(\Delta F)$ was defined as the difference of $F_{0}$ and $F_{t}$.

\section{Results and discussion}

\subsection{Optimization of experimental conditions}

In this study, sodium citrate, carbamide and cobalt chloride were adopted as precursors for the synthesis of CQDs, while methylbenzene was selected as solvent. It is reported that mass fraction of precursors, reaction temperature and reaction time have great influence on the fluorescence properties of carbon quantum dots. ${ }^{59}$ In order to prepare CQDs with strong photoluminescence intensity, the above three reaction parameters were systematically determined by a series of experiments. Firstly, the influence of mass fraction of cobalt chloride on the fluorescence intensity was studied, which is demonstrated in Fig. 1(a). It can be found that maximum fluorescence intensity $\left(1.02086 \times 10^{6}\right)$ of CQDs was attained, when the proportion (molar ratio) of the sodium citrate, carbamide and cobalt chloride is $1: 2: 1.5$ (the reaction temperature: $180{ }^{\circ} \mathrm{C}$, the reaction time: $24 \mathrm{~h}$ ). The effect of reaction temperature on fluorescence intensity is also showed in Fig. 1(b). A significant increase of fluorescence intensity was obtained between 140 and $180{ }^{\circ} \mathrm{C}$. When the temperature increased from 180 to $220^{\circ} \mathrm{C}$, the fluorescence intensity showed a decreasing trend. As a result, $180{ }^{\circ} \mathrm{C}$ is the optimum reaction temperature for the synthesis of CQDs, when the proportion (molar ratio) of the sodium citrate, carbamide and cobalt chloride is $1: 2: 1.5$ and the reaction time is $24 \mathrm{~h}$. Besides, the influence of the reaction time on fluorescence intensity was investigated, when the proportion (molar ratio) of the sodium citrate, carbamide and cobalt chloride is fixed at $1: 2: 1.5$ and the reaction temperature is $180{ }^{\circ} \mathrm{C}$. According to Fig. $1(\mathrm{c})$, it is found that the optimum reaction time is $24 \mathrm{~h}$. The absolute fluorescence quantum yield of the as-prepared carbon quantum dots was measured by integration sphere of FS5. The result shows that the absolute fluorescence quantum yields is $92.6 \%$.

\subsection{The morphology characterization of carbon quantum dots}

The morphology of CQDs synthesized under optimum experimental conditions was visualized by the transmission electron microscopy (TEM). It can be seen from the TEM image (Fig. 2(a)) that the shape of the as-prepared CQDs is spherical and disperse evenly. The high-resolution TEM (HRTEM) images (the inset of Fig. 2(a)) revealed the high crystallinity of CQDs. The lattice spacing of $0.21 \mathrm{~nm}$ is in agreement with that of the inplane lattice spacing of graphene (100 facet) and the spacing between graphite layers (100 facet). ${ }^{60}$ In addition, the Fig. $2(\mathrm{~b})$ is the particle size distribution histogram of Fig. 2(a), which shows 
(a)

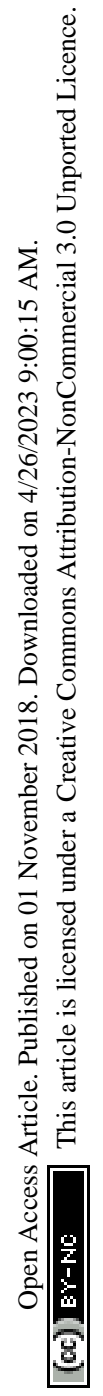

(b)

(c)
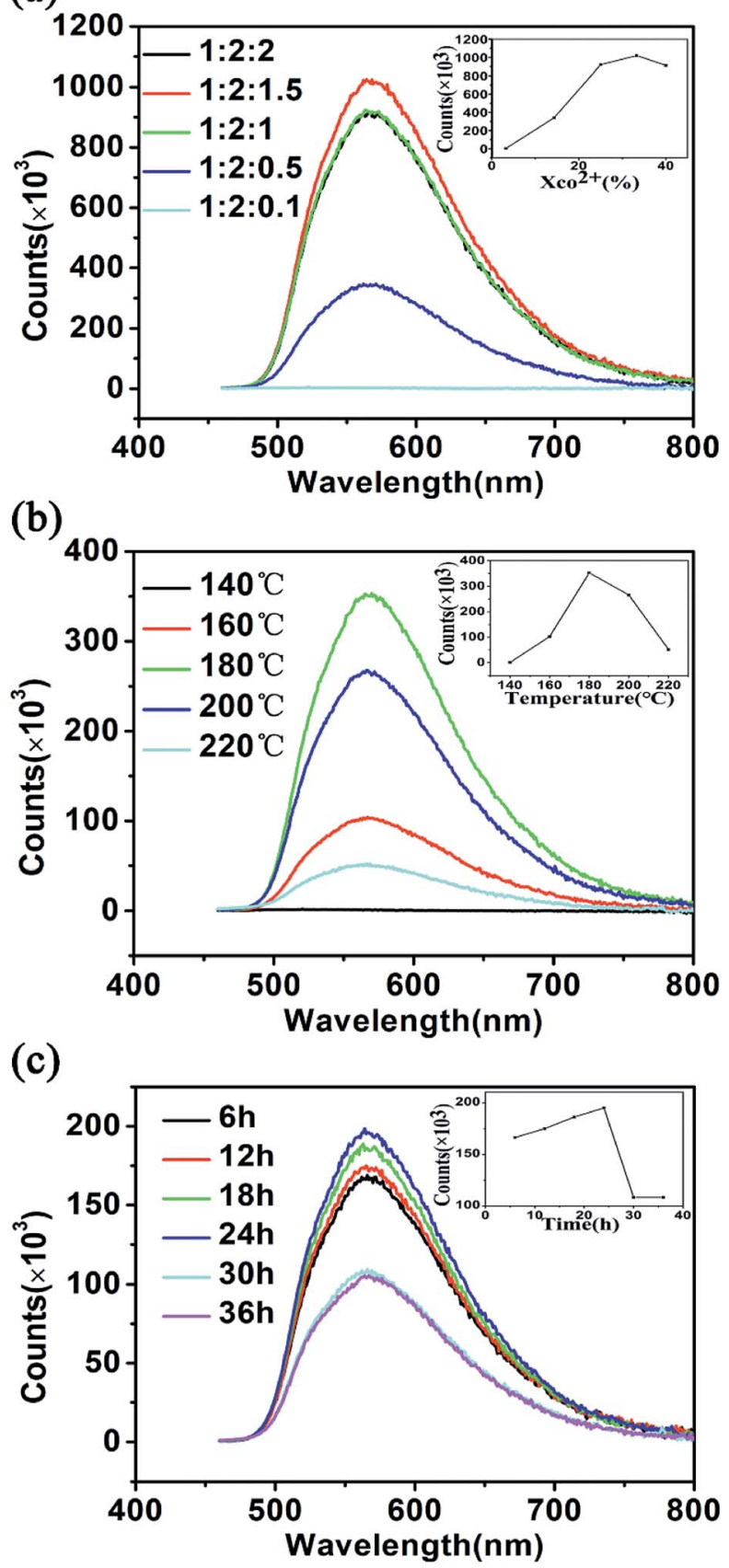

Fig. 1 Effects of (a) mass fraction of cobalt chloride, (b) reaction temperature, and (c) reaction time on the fluorescence intensity.

that the diameter of the as-prepared CQDs is distributed in the range from 3.3 to $8.1 \mathrm{~nm}$ with an average size of $5.2 \mathrm{~nm}$.

\subsection{The structure characterization of carbon quantum dots}

The FTIR (Fourier transform infrared spectroscopy) and XPS (Xray photoelectron spectroscopy) were adopted to further characterize functional groups and chemical bound of CQDs. As shown in Fig. 3(a), the CQDs show three strong peaks at 1708, 1258 , and $1009 \mathrm{~cm}^{-1}$, which attributed to $\mathrm{C}=\mathrm{O}, \mathrm{C}-\mathrm{N}$ and $\mathrm{O}-\mathrm{H}$ stretching vibration, respectively. ${ }^{61}$ What' more, the peak at around $3000-2850 \mathrm{~cm}^{-1}$ is ascribed to the $\mathrm{C}-\mathrm{H}$ stretching

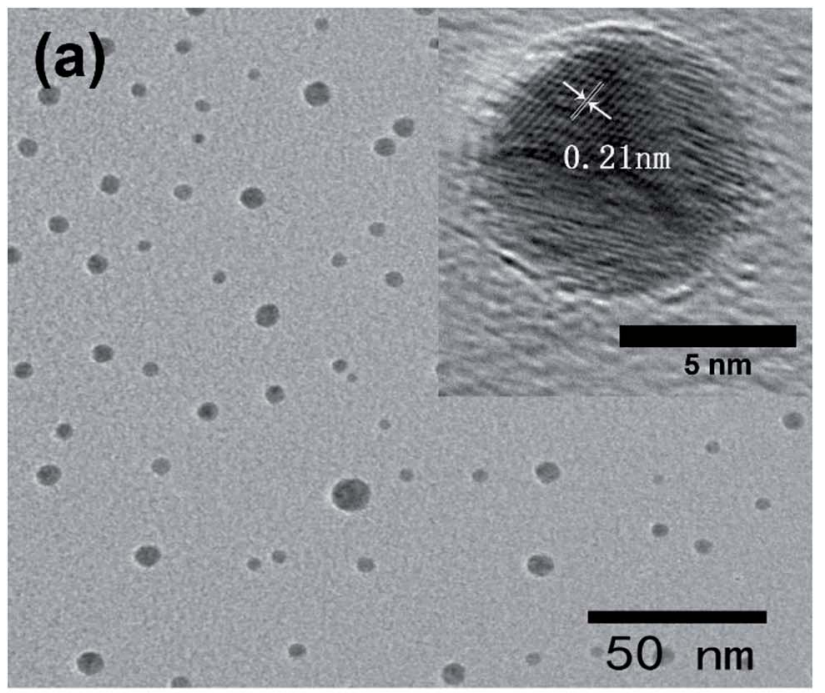

(b)

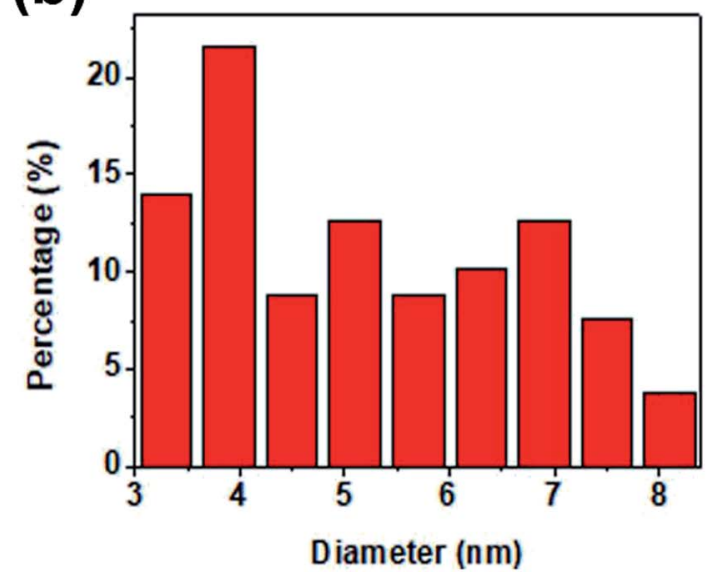

Fig. 2 (a) The TEM image of CQDs (the inset shows the high resolution TEM images of single CQD), (b) the corresponding particle size distribution histogram of (a).

vibration. ${ }^{62}$ The broad band at $800-600 \mathrm{~cm}^{-1}$ corresponds to the $\mathrm{N}-\mathrm{H}$ bending vibration. ${ }^{63}$ Moreover, the surface components and oxidation states of CQDs were also characterized by XPS. The Fig. 3(b) shows four peaks centred at $284.48 \mathrm{eV}, 400.22 \mathrm{eV}$, and $532.24 \mathrm{eV}$, which correspond with $\mathrm{C}_{1 \mathrm{~s}}, \mathrm{~N}_{1 \mathrm{~s}}$, and $\mathrm{O}_{1 \mathrm{~s}}$ respectively. The result suggestion that the as-prepared CQDs contained $\mathrm{C}, \mathrm{N}, \mathrm{O}$ with $\%$ atom ratio of $\mathrm{C}: \mathrm{N}: \mathrm{O}=$ $77.58: 4.55: 17.86$. The high-resolution spectra of $\mathrm{C}_{1 \mathrm{~s}}, \mathrm{~N}_{1 \mathrm{~s}}$ were demonstrated in Fig. 3(c) and (d). The high-resolution spectrum of $\mathrm{C}_{1 \mathrm{~s}}$ show four peaks at $284.58 \mathrm{eV}, 286.68 \mathrm{eV}, 288.38 \mathrm{eV}$ and $285.28 \mathrm{eV}$, which correspond with $\mathrm{C}-\mathrm{C}, \mathrm{C}-\mathrm{O}, \mathrm{C}=\mathrm{O}$ and $\mathrm{C}-\mathrm{N}$ respectively. The high-resolution spectrum of $\mathrm{N}_{1 \mathrm{~s}}$ can be deconvoluted into three peaks, $\mathrm{C}-\mathrm{NH}_{2}(399.08 \mathrm{eV})$, pyridinic-N $(400.38 \mathrm{eV})$ and pyrrolic-N (399.98 eV). Fig. 3(e) shows the UVvis absorption spectrum of as-prepared CQDs. The absorption peak at $290 \mathrm{~nm}$ is ascribed to the formation of aromatic $\pi$ orbitals, due to the formation of graphitic carbon structure. What's more, there is another absorption peak in $420 \mathrm{~nm}$ in the UV-vis absorption spectrum for CQDs, which corresponds with the excitation spectrum (Fig. 3(f)). From the results, we can infer 
(a)

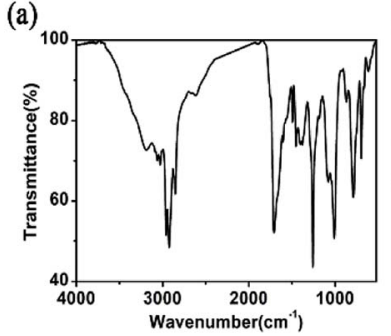

(c)

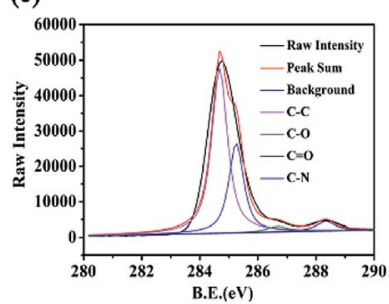

(e)

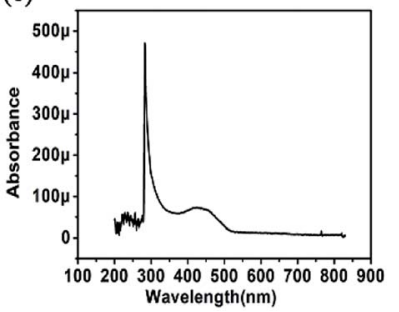

(b)

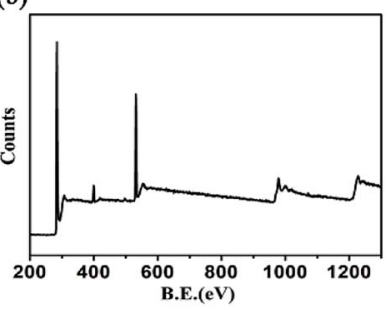

(d)

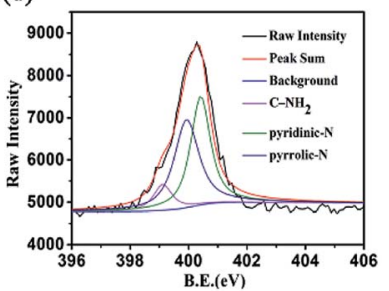

(f)

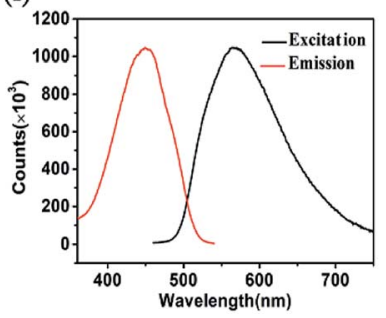

Fig. 3 (a) The FTIR spectrum of the as-prepared CQDs; (b) the survey XPS spectrum of the as-prepared QDs; (c) high-resolution XPS spectrum of $\mathrm{C}_{1 \mathrm{~s}}$ of the as-prepared CQDs; (d) high-resolution XPS spectrum of $\mathrm{N}_{1 \mathrm{~s}}$ of the as-prepared CQDs; (e) the UV-vis absorption spectrum of the as-prepared CQDs; ( $f$ ) the fluorescence excitation spectrum and emission spectrum.

that the CQDs are evenly distributed. In other words, the asprepared CQDs had identical absorption structure and luminescent centre.

\subsection{Optical properties of carbon quantum dots}

Fig. 4 demonstrated the 3D map (Fig. 4(a)), contour plot (Fig. 4(b)) and the emission spectra (Fig. 4(c)) excited by the light of wavelength range from 300 to $500 \mathrm{~nm}$ of the as-prepared CQDs. According to the comparison and analysis of Fig. 4(a)-(c), it can be found that when the excited at $\sim 440 \mathrm{~nm}$ the asprepared CQDs have the strongest emission at $570 \mathrm{~nm}$. The toluene solution of CQDs exhibits orange-yellow color (left inset, Fig. 4(c)) in day light and bright yellow emission under UV light (365 nm) (right inset in Fig. 4(c)). What's more, the CQDs exhibit excitation independent emission properties, due to the homogeneous surface structure, and monodispersity (Fig. 1(a)) of CQDs. What is more, it is found that the fluorescent properties of CQDs are excellent and stable in organic solvents (toluene, ethanol, 1,4-dioxane, tetrahydrofuran) (Fig. S1†).

\subsection{Fluorescence "turn-off" determination of water content in organic solvents}

Water exists in all kinds of organic solvents and is the most common impurity in organic solvents. The water content in the
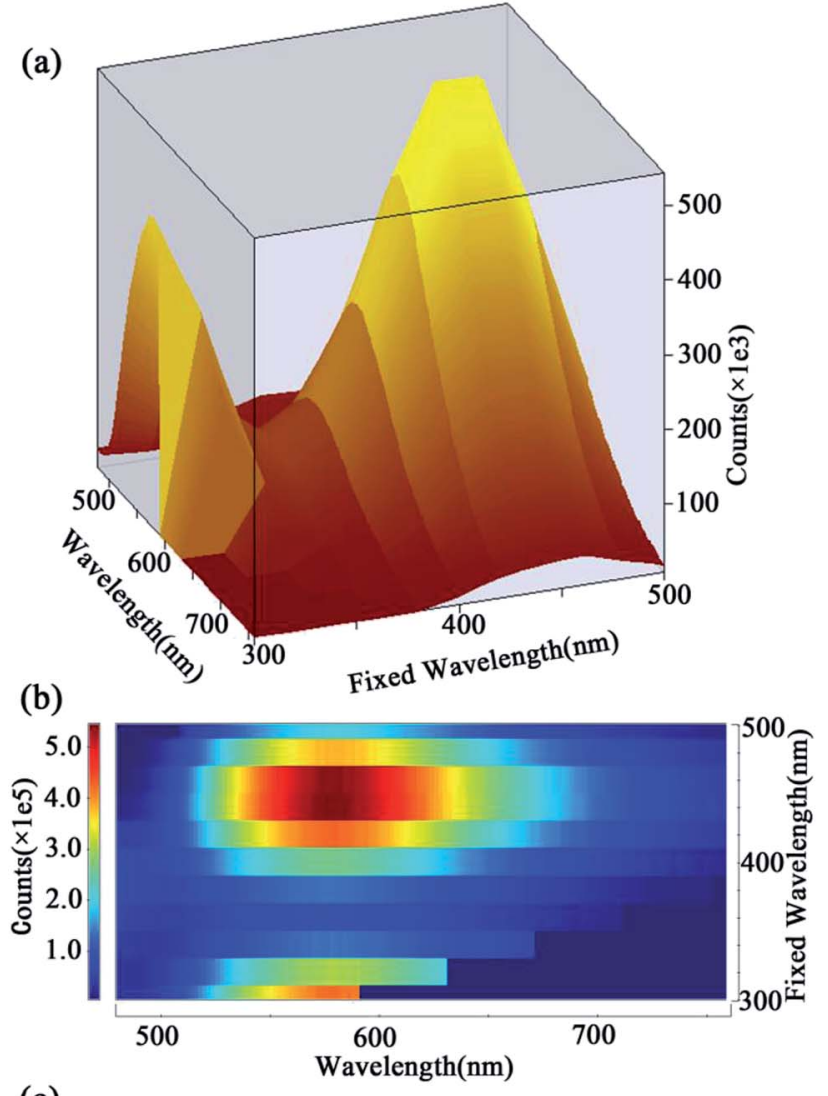

(c)

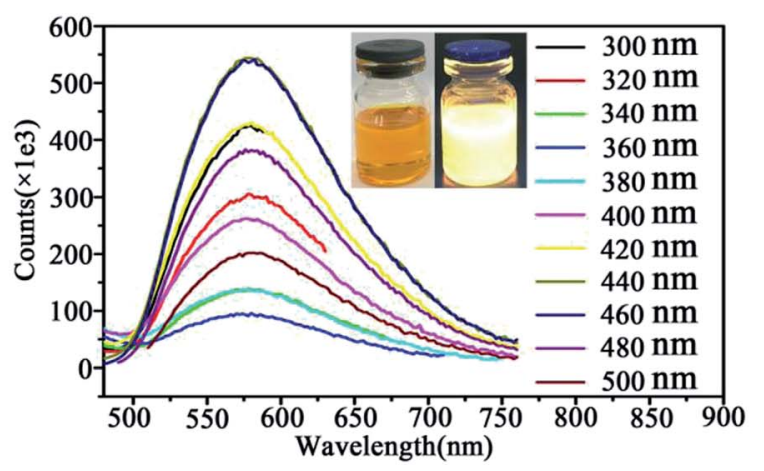

Fig. 4 (a) The fluorescence emission-excitation 3D map of the asprepared CQDs; (b) the contour plot of photoluminescence spectrum for CQDs; (c) photoluminescence spectrum of the as-prepared CQDs (inset: photographs of CQDs toluene solutions exposed to daylight (left) and $365 \mathrm{~nm}$ UV radiation (right)).

organic solvent will influence the chemical reaction to a great extent, and even determine the product of the reaction. Therefore, the determination of the water content in the organic solvent is one of the most important and most commonly encountered analytical problems. ${ }^{2,3,5,7,13}$

In order to explore the possibility for application of the asprepared CQDs in the detection of water content in organic solvents, the fluorescence emission spectra excited by the light of $440 \mathrm{~nm}$ of CQDs dispersed in ethanol were further examined. As shown in Fig. $5(\mathrm{a})$, when $10 \% \mathrm{v} / \mathrm{v}$ water was added into the ethanol solution of CQDs, the fluorescent intensity decreased significantly for one minute and then tends to be stable. This 
indicates that CQDs can be used as fluorescent probes to quickly detect water content in organic solvents. Fig. 5(b) shows the fluorescence emission spectra of CQDs dispersed in ethanol with different concentration of $\mathrm{H}_{2} \mathrm{O}(0,0.01,2,4,6,8,10,20,50$, $80,100 \mathrm{v} / \mathrm{v}, \%)$. It can be seen that with the increase of water content, the fluorescence intensity decreases gradually and the emission peak remain the same. Fig. 5(c) shows that the fluorescent intensity decreased with the addition of water in the ethanol solution of CQDs. It can be found that when the water content exceeds $10 \%$, the decrease of fluorescence intensity gradually slows. The resulting calibration curve for $\mathrm{H}_{2} \mathrm{O}$ showed good linearity for the range of concentrations from $0.01 \%(\mathrm{v} / \mathrm{v})$ to $10 \%(\mathrm{v} / \mathrm{v})$ with a correlation coefficient of 0.9970 (Fig. $5(\mathrm{~d})$ ). The limit of detection was as low as $0.01 \%$ based on five times the standard deviation rule. The limit of detection is lower than that of those previously reported..$^{\mathbf{6 1 2 , 6 4 - 6 7}}$ Furthermore, the water content in tetrahydrofuran, 1,4-dioxane is also rapidly detected using the as-prepared CQDs (see details in Fig. S2 and S3†).

\subsection{The possible mechanism for trace water detection in organic solvents}

In order to explore the mechanism for the detection of water content in organic solvents, the fluorescence emission spectra excited by the light of $440 \mathrm{~nm}$ of CQDs dispersed in different organ solvents (toluene, tetrahydrofuran, 1,4-dioxane, ethanol) with increasing polarity were further examined. As shown in Fig. 6(a), the emission peak of CQDs dispersed in toluene, tetrahydrofuran, 1,4-dioxane and ethanol shifted to undergoes red shift with increasing solvent polarity. What is more, the fluorescent intensity decreases with increase of solvent polarity, which conform to solvation effect. The above-mentioned
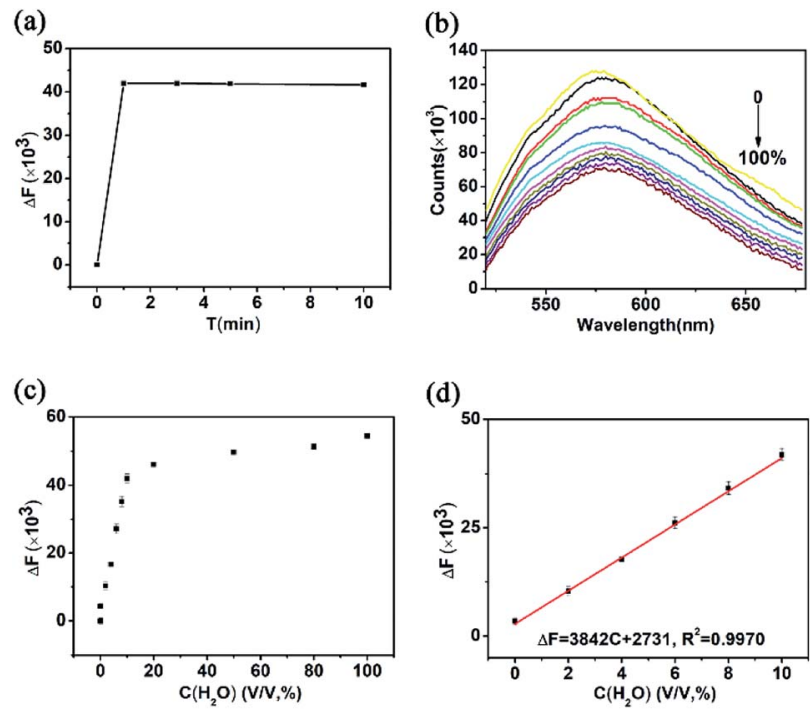

Fig. 5 (a) Time-dependent fluorescence quenching of CQDs dispersed in ethanol in the presence of $\mathrm{H}_{2} \mathrm{O}(10 \%, \mathrm{v} / \mathrm{v})$; (b) the fluorescence emission spectra of CQDs dispersed in ethanol with different concentration of $\mathrm{H}_{2} \mathrm{O}$ $(0,0.01,2,4,6,8,10,20,50,80,100 \mathrm{v} / \mathrm{v}, \%)$; (c) the change of fluorescence intensity of CQDs solution versus the concentration of $\mathrm{H}_{2} \mathrm{O}$ from $0.01 \%$ to $100 \%$; (d) a linear relationship between $\Delta F$ and concentration of $\mathrm{H}_{2} \mathrm{O}$ from $0.01 \%$ to $10 \%$. Error bars in (c) and (d) represent the standard deviations of five independent measurements. (a)

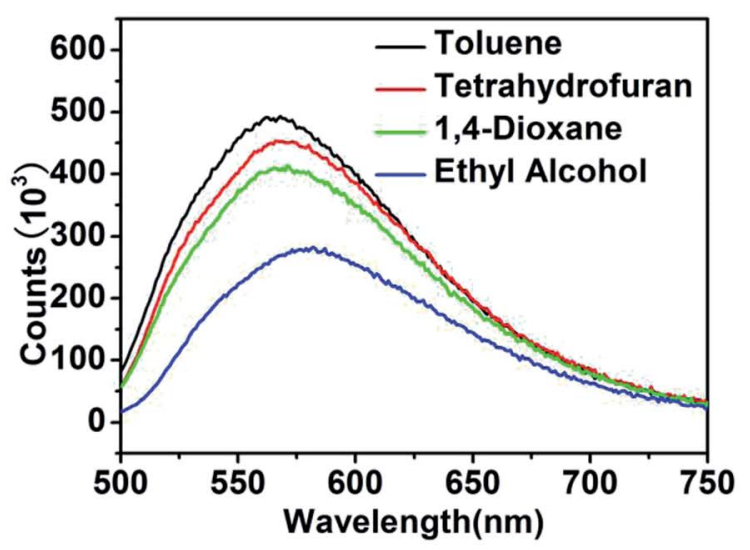

(b)

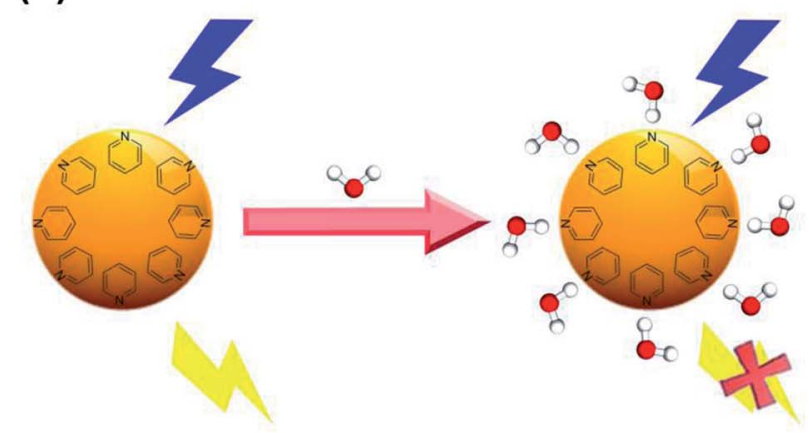

Fig. 6 (a) The fluorescence emission spectra (excited by the light of $440 \mathrm{~nm}$ ) of CQDs dispersed in different organ solvents (toluene, tetrahydrofuran, 1,4-dioxane, ethanol); (b) schematic illustration for the detection of $\mathrm{H}_{2} \mathrm{O}$ in organic solvents by CQDs.

fluorescent property is consistent with that of internal charge transfer (ICT) fluorescent probes. ${ }^{68,69}$ According to Fig. 5, S1 and $\mathrm{S} 2, \dagger$ it can be found that the emission peak of CQDs in tetrahydrofuran and 1,4-dioxane shifted to higher wavelength with the increase of the $\mathrm{H}_{2} \mathrm{O}$ concentration, while the emission peak of CQDs in ethanol showed no remarkable change with the increase of the $\mathrm{H}_{2} \mathrm{O}$ concentration. This, combined with the results of Fig. 6(a), indicates that the presence of hydroxyl in ethanol and water $(-\mathrm{OH})$ will lead to the red-shift effect for the emission peak and decrease of fluorescence intensity. According to the results of XPS, there is pyridinic-N in the structure of CQDs. Because the electron density around pyridinic- $\mathrm{N}$ is higher, this atom serves as a stronger acceptor of hydrogen bonds, which increases the possibility of nonradiative relaxation of the excited CQDs and leads to the decrease of fluorescent intensity in the presence of hydrogen bond donating molecules, such as ethyl alcohol and water. ${ }^{70}$ In a word, the decrease of fluorescence intensity of asprepared CQDs is attributed to the specific water-fluorophore interaction and partially to the increase in polarity of the solvent caused by the addition of water.

\section{Conclusions}

In summary, homogeneous carbon quantum dots with yellow fluorescence and high PL were firstly prepared by solvothermal 
method adopting sodium citrate, carbamide, and cobalt chloride as precursors with toluene as solvent. The as-prepared CQDs with high crystallinity have spherical shape with an average size of $5.2 \mathrm{~nm}$. It is found that the fluorescent properties of CQDs are excellent in organic solvents and could be quenched by trace water, which makes them a promising material used without any modification for the detection of water in organic solvents. The as-prepared CQDS were adopted as fluorescent probes for the detection of trace water organic solvents according to the luminescence response with correlation coefficient of 0.9983 (in ethanol), 0.9990 (in tetrahydrofuran), 0.9963 (in 1,4-dioxane), respectively. This is the first time that CQDs were adopted as the water sensing fluorescent probe. This work makes the CQDs a promising material used without any modification in the detection of water in organic solvents and with immense potential for applications in quality management of the solvent products. The possible mechanism for trace water detection in organic solvents is attributed to the specific water-fluorophore interaction and partially to the increase in polarity of the solvent caused by the increase of water concentration.

\section{Conflicts of interest}

There are no conflicts to declare.

\section{References}

1 M. Bai and W. R. Seitz, Talanta, 1994, 41, 993-999.

2 J. Blyth, R. B. Millington, A. G. Mayes, E. R. Frears and C. R. Lowe, Anal. Chem., 1996, 68, 1089-1094.

3 U. Fegade, S. Patil, R. Kaur, S. K. Sahoo, N. Singh, R. Bendre and A. Kuwar, Sens. Actuators, B, 2015, 210, 324-327.

4 D.-C. Han, Y.-J. Jin, J.-H. Lee, S.-I. Kim, H.-J. Kim, K.-H. Song and G. Kwak, Macromol. Chem. Phys., 2014, 215, 1068-1076.

5 H. S. Jung, P. Verwilst, W. Y. Kim and J. S. Kim, Chem. Soc. Rev., 2016, 45, 1242-1256.

6 K.-H. Kim and H. J. Kim, Bull. Korean Chem. Soc., 2015, 36, 183-188.

7 W. Y. Kim, H. Shi, H. S. Jung, D. Cho, P. Verwilst, J. Y. Lee and J. S. Kim, Chem. Commun., 2016, 52, 8675-8678.

8 Z. Li, Q. Yang, R. Chang, G. Ma, M. Chen and W. Zhang, Dyes Pigm., 2011, 88, 307-314.

9 G. Men, C. Chen, C. Liang, W. Han and S. Jiang, Analyst, 2015, 140, 5454-5458.

10 G. Men, G. Zhang, C. Liang, H. Liu, B. Yang, Y. Pan, Z. Wang and S. Jiang, Analyst, 2013, 138, 2847-2857.

11 Y. Ooyama, S. Aoyama, K. Furue, K. Uenaka and J. Ohshita, Dyes Pigm., 2015, 123, 248-253.

12 Y. Ooyama, K. Uenaka, A. Matsugasako, Y. Harima and J. Ohshita, RSC Adv., 2013, 3, 23255-23263.

13 F. Opekar and P. Tůma, Sens. Actuators, B, 2015, 220, 485490.

14 S. Pal, M. Mukherjee, B. Sen, S. Lohar and P. Chattopadhyay, RSC Adv., 2014, 4, 21608-21611.

15 H.-S. Peng, X.-H. Li, F.-T. You, F. Teng and S.-H. Huang, Microchim. Acta, 2013, 180, 807-812.
16 A. C. Pereira, B. F. Reis and F. R. P. Rocha, Talanta, 2015, 131, 21-25.

17 Y. Sun, X. Liang, S. Wei, J. Fan and X. Yang, Spectrochim. Acta, Part A, 2012, 97, 352-358.

18 J. Qi, B. Li, X. Wang, Z. Zhang, Z. Wang, J. Han and L. Chen, Sens. Actuators, B, 2017, 251, 224-233.

19 M. Jia, Z. Zhang, J. Li, H. Shao, L. Chen and X. Yang, Sens. Actuators, B, 2017, 252, 934-943.

20 J. Yu, X. Wang, Q. Kang, J. Li, D. Shen and L. Chen, Environ. Sci.: Nano, 2017, 4, 493-502.

21 X.-R. Wang, B.-W. Li, H.-Y. You and L.-X. Chen, Chin. J. Anal. Chem., 2015, 43, 1499-1504.

22 J. Zhang, J. Wang, J. Fu, X. Fu, W. Gan and H. Hao, J. Nanopart. Res., 2018, 20, 41.

23 W. Yang, H. Zhang, J. Lai, X. Peng, Y. Hu, W. Gu and L. Ye, Carbon, 2018, 128, 78-85.

24 J. Xu, Y. Miao, J. Zheng, H. Wang, Y. Yang and X. Liu, Nanoscale, 2018, 10, 11211-11221.

25 Q. Wu, X. Wang, S. A. Rasaki, T. Thomas, C. Wang, C. Zhang and M. Yang, J. Mater. Chem. C, 2018, 6, 4508-4515.

26 Z. Miao, W. Hou, M. Liu, Y. Zhang and S. Yao, New J. Chem., 2018, 42, 1446-1456.

27 Z. Li, J. Zhang, Y. Li, S. Zhao, P. Zhang, Y. Zhang, J. Bi, G. Liu and Z. Yue, Biosens. Bioelectron., 2018, 99, 251-258.

28 R. Wang, X. Wang and Y. Sun, Sens. Actuators, B, 2017, 241, 73-79.

29 R. Wang, K.-Q. Lu, Z.-R. Tang and Y.-J. Xu, J. Mater. Chem. A, 2017, 5, 3717-3734.

30 A. Vassilakopoulou, V. Georgakilas, N. Vainos and I. Koutselas, J. Phys. Chem. Solids, 2017, 103, 190-196.

31 L. Sciortino, F. Messina, G. Buscarino, S. Agnello, M. Cannas and F. M. Gelardi, J. Nanopart. Res., 2017, 19, 228.

32 W. Liu, C. Li, X. Sun, W. Pan and J. Wang, Sens. Actuators, B, 2017, 244, 441-449.

33 X. Cui, Y. Wang, J. Liu, Q. Yang, B. Zhang, Y. Gao, Y. Wang and G. Lu, Sens. Actuators, B, 2017, 242, 1272-1280.

34 B. Zhu, C. Shang and Z. Guo, ACS Sustainable Chem. Eng., 2016, 4, 1050-1057.

35 Y. H. Yuan, Z. X. Liu, R. S. Li, H. Y. Zou, M. Lin, H. Liu and C. Z. Huang, Nanoscale, 2016, 8, 6770-6776.

36 L. Xu, G. Fang, M. Pan, X. Wang and S. Wang, Biosens. Bioelectron., 2016, 77, 950-956.

37 N. Wang, Y. Wang, T. Guo, T. Yang, M. Chen and J. Wang, Biosens. Bioelectron., 2016, 85, 68-75.

38 L. Wang, B. Li, F. Xu, X. Shi, D. Feng, D. Wei, Y. Li, Y. Feng, Y. Wang, D. Jia and Y. Zhou, Biosens. Bioelectron., 2016, 79, 1-8.

39 S. Y. Park, H. U. Lee, E. S. Park, S. C. Lee, J.-W. Lee, S. W. Jeong, C. H. Kim, Y.-C. Lee, Y. S. Huh and J. Lee, ACS Appl. Mater. Interfaces, 2014, 6, 3365-3370.

40 T. Feng, X. Ai, G. An, P. Yang and Y. Zhao, ACS Nano, 2016, 10, 4410-4420.

41 F. Wang, Z. Xie, H. Zhang, C.-y. Liu and Y.-g. Zhang, Adv. Funct. Mater., 2011, 21, 1027-1031.

42 S. Yang, L. Zhi, K. Tang, X. Feng, J. Maier and K. Müllen, Adv. Funct. Mater., 2012, 22, 3634-3640. 
43 L. Bao, Z.-L. Zhang, Z.-Q. Tian, L. Zhang, C. Liu, Y. Lin, B. Qi and D.-W. Pang, Adv. Mater., 2011, 23, 5801-5806.

44 B. Kong, A. Zhu, C. Ding, X. Zhao, B. Li and Y. Tian, Adv. Mater., 2012, 24, 5844-5848.

45 J. Liang, Y. Jiao, M. Jaroniec and S. Z. Qiao, Angew. Chem., Int. Ed. Engl., 2012, 51, 11496-11500.

46 S. Qu, X. Wang, Q. Lu, X. Liu and L. Wang, Angew. Chem., Int. Ed. Engl., 2012, 51, 12215-12218.

47 S. Zhu, Q. Meng, L. Wang, J. Zhang, Y. Song, H. Jin, K. Zhang, H. Sun, H. Wang and B. Yang, Angew. Chem., Int. Ed. Engl., 2013, 52, 3953-3957.

48 W. Wu, L. Zhan, W. Fan, J. Song, X. Li, Z. Li, R. Wang, J. Zhang, J. Zheng, M. Wu and H. Zeng, Angew. Chem., 2015, 127, 6640-6644.

49 A. B. Bourlinos, M. A. Karakassides, A. Kouloumpis, D. Gournis, A. Bakandritsos, I. Papagiannouli, P. Aloukos, S. Couris, K. Hola, R. Zboril, M. Krysmann and E. P. Giannelis, Carbon, 2013, 61, 640-643.

50 X. Ren, J. Liu, X. Meng, J. Wei, T. Liu and F. Tang, Chem. Asian J., 2014, 9, 1054-1059.

51 M. X. Gao, C. F. Liu, Z. L. Wu, Q. L. Zeng, X. X. Yang, W. B. Wu, Y. F. Li and C. Z. Huang, Chem. Commun., 2013, 49, 8015-8017.

52 H. Zhu, X. Wang, Y. Li, Z. Wang, F. Yang and X. Yang, Chem. Commun., 2009, 5118-5120, DOI: 10.1039/b907612c.

53 V. Gupta, N. Chaudhary, R. Srivastava, G. D. Sharma, R. Bhardwaj and S. Chand, J. Am. Chem. Soc., 2011, 133, 9960-9963.

54 R. Ye, C. Xiang, J. Lin, Z. Peng, K. Huang, Z. Yan, N. P. Cook, E. L. Samuel, C. C. Hwang, G. Ruan, G. Ceriotti, A. R. Raji, A. A. Marti and J. M. Tour, Nat. commun., 2013, 4, 2943.

55 Y. Ma, G. Xu, F. Wei, Y. Cen, Y. Ma, Y. Song, X. Xu, M. Shi, S. Muhammad and Q. Hu, J. Mater. Chem. C, 2017, 5, 8566-8571.
56 M. Amjadi and R. Jalili, Biosens. Bioelectron., 2017, 96, 121126.

57 A. A. Ensafi, P. Nasr-Esfahani and B. Rezaei, Sens. Actuators, $B, 2018,257$, 889-896.

58 N. Yu, H. Peng, H. Xiong, X. Wu, X. Wang, Y. Li and L. Chen, Microchim. Acta, 2015, 182, 2139-2146.

59 Q. Xu, Y. Liu, C. Gao, J. Wei, H. Zhou, Y. Chen, C. Dong, T. S. Sreeprasad, N. Li and Z. Xia, J. Mater. Chem. C, 2015, 3, 9885-9893.

60 C. J. Reckmeier, J. Schneider, A. S. Susha and A. L. Rogach, Opt. Express, 2016, 24, A312-A340.

61 X. Meng, J. Wei, X. Ren, J. Ren and F. Tang, Biosens. Bioelectron., 2013, 47, 402-407.

62 H. Li, X. He, Y. Liu, H. Huang, S. Lian, S.-T. Lee and Z. Kang, Carbon, 2011, 49, 605-609.

63 P. C. Hsu and H. T. Chang, Chem. Commun., 2012, 48, 39843986.

64 K. Zhao, T. Liu, G. Wang, X. Chang, D. Xue, K. D. Belfield and Y. Fang, J. Phys. Chem. B, 2013, 117, 5659-5667.

65 Y. Ooyama, M. Sumomogi, T. Nagano, K. Kushimoto, K. Komaguchi, I. Imae and Y. Harima, Org. Biomol. Chem., 2011, 9, 1314-1316.

66 Y. Ooyama, A. Matsugasako, K. Oka, T. Nagano, M. Sumomogi, K. Komaguchi, I. Imae and Y. Harima, Chem. Commun., 2011, 47, 4448-4450.

67 Q. Deng, Y. Li, J. Wu, Y. Liu, G. Fang, S. Wang and Y. Zhang, Chem. Commun., 2012, 48, 3009-3011.

68 M. H. Lee, J. S. Kim and J. L. Sessler, Chem. Soc. Rev., 2015, 44, 4185-4191.

69 A. P. de Silva, H. Q. N. Gunaratne, T. Gunnlaugsson, A. J. M. Huxley, C. P. McCoy, J. T. Rademacher and T. E. Rice, Chem. Rev., 1997, 97, 1515-1566.

70 D. Citterio, K. Minamihashi, Y. Kuniyoshi, H. Hisamoto, S.-I. Sasaki and K. Suzuki, Anal. Chem., 2001, 73, 5339-5345. 